\title{
La mediazione in fase esecutiva nel sistema italiano: il quadro normativo e le dinamiche applicative
}

\author{
The victim-offender mediation in the post-sentencing stage within \\ the Italian system: an overview on legal framework and case law
}

\section{Daniele Vicoli ${ }^{1}$}

Alma Mater Studiorum - Università di Bologna, Bologna, Italia

daniele.vicoli@unibo.it

https://orcid.org/0000-0002-4505-4147

\begin{abstract}
AвSTRACT: Nel sistema italiano, la vittima, sebbene abbia assunto un ruolo di crescente importanza, resta ai margini della fase di esecuzione della pena. All'indifferenza legislativa se ne aggiunge un'altra: il tema della mediazione - al centro di diffuse analisi sul piano delle possibili alternative al rito ordinario - risulta esaminato in modo superficiale nel quadro delle dinamiche esecutive. L'articolo intende offrire un contributo utile a colmare questa lacuna. Nelle linee di fondo, il tratto distintivo della mediazione in executivis è rappresentato dall'intervenuta irrevocabilità della sentenza: un fattore che si palesa ambivalente, nella misura in cui può agevolare percorsi a valenza conciliativa ma anche renderli più ostici. In simile scenario, diventa centrale il nesso tra la giustizia riparativa e gli scopi di risocializzazione sottesi alla pena, tali da esplicarsi nell'impegno dell'autore a rivisitare in chiave critica l'illecito commesso e ricostruire il rapporto con la persona offesa. Stabilita questa premessa, l'accento va posto sulla logica del dialogo: le parti sono chiamate a sviluppare, con l'aiuto del mediatore, una trama relazionale che permetta di sanare la frattura originata dal reato. Tali canoni - già sfuggenti nella dimensione normativa dell'affidamento in prova (art. 47 comma 7 ord. penit.) e del lavoro all'esterno (art. 21 comma 4-ter ord. penit.) - sono del tutto obliterati sul versante applicativo. Appare, quindi, indispensabile un
\end{abstract}

1 Professore associato di Diritto processuale penale - Alma Mater Studiorum Università di Bologna. 
deciso cambio di rotta al fine di introdurre, nella fase esecutiva, forme di mediazione a carattere individualizzato e comunicativo.

Parole ChIAVE: Esecuzione penale; mediazione; giustizia riparativa; affidamento in prova; lavoro all'esterno.

ABSTRACT: Within the Italian criminal justice system, the role of the victim has become increasingly important. Despite this trend, the victim still stands on the edge of the post-sentencing stage. This is reflected in the fact that the topic of the victim-offender mediation has been analysed deeply as a kind of diversion to the trial but not, or at least far less, in relation to the issues concerning the enforcement of penalty. The paper is aimed at filling this gap. As a starting point, it is to be highlighted that the irrevocability of the conviction poses advantages but also critical aspects in the perspective of reconciliation. In this context, surely the effort of the convicted person to review his/her criminal past critically and rebuild his/her relationships with the victim plays a central role. However, according to the restorative justice model, an interaction between the victim and the offender shall take place. This means that both parties have to assume a proactive role in the attempt of mutually rectifying, with the assistance of a mediator, the conflict originated by the commission of the offence. The analysis of both the provisions and the case law on probation (article 47 comma 7 ord. penit.) and volunteer work (art. 21 comma 4-ter ord. penit.) points out that the mentioned key-principles are not abided. Against this background, a radical change is proposed in order to introduce a mediation model based on individualized and relational elements in the post-sentencing stage.

KEYWORDS: Enforcement of penalty; victim-offender mediation; restorative justice; probation; volunteer work.

Sommarıo: 1. Premessa: gli angusti spazi normativi; 2. La mediazione nella fase esecutiva: tratti distintivi; 3. Un quadro ambivalente: vantaggi e ostacoli; 4. II nesso con il finalismo rieducativo della pena; 5. Un panorama applicativo deludente; 6. Conclusioni; Bibliografia.

\section{Premessa: gli angusti spazi normativi.}

Sono molteplici gli interrogativi che circondano l'idea di calare, all'interno della fase esecutiva, modelli ascrivibili al paradigma della 
mediazione $^{2}$ : le distanze sembrerebbero tali da rendere impraticabile ogni forma di osmosi. In questo senso, non è un caso - potrebbe sostenersi - che la dottrina, almeno in Italia, abbia mostrato per il tema un interesse solo parziale, soprattutto se il termine di paragone sono le diffuse analisi dedicate ai rapporti tra il procedimento di cognizione e gli istituti a valenza mediativa.

Anche la crescente valorizzazione del ruolo da riconoscere alla vittima ha lasciato labili tracce. Come testimonia un rapido sguardo d'insieme, permane una diffusa indifferenza: la persona offesa è un «vero "convitato di pietra" $»^{3}$ a fronte degli snodi nei quali si articola l'esecuzione della pena.

Il panorama normativo è apparso incurante delle molteplici istanze che si collocano nell'alveo delle dinamiche riparative, suscettibili di essere sviluppate secondo una pluralità di schemi: il tratto unitario si rinviene nella spinta a instaurare, allo scopo di favorire la ricerca di una ritrovata armonia, relazioni comunicative tra le parti antagoniste del conflitto la cui genesi è dovuta alla commissione del reato ${ }^{4}$.

In quest'ottica, sul piano delle ricadute interne, anche la direttiva 29/2012/UE ${ }^{5}$ si è rivelata sterile: nel recepirla, il legislatore italiano non

2 La Raccomandazione (99) 19 del Comitato dei Ministri del Consiglio d'Europa definisce la mediazione «qualsiasi processo nell'ambito del quale la vittima e l'autore di un reato sono messi in condizione, se vi acconsentono liberamente, di partecipare alla soluzione delle questioni derivanti da un reato attraverso l'aiuto di un terzo imparziale (il mediatore)». Per un inquadramento di carattere generale, v. CASTELLI, Stefano. La mediazione. Teorie e tecniche, Milano: Raffaello Cortina, 1996, p. 5; MANNOZZI, Grazia; LODIGIANI, Giovanni Angelo. La giustizia riparativa, Torino: Giappichelli, 2017, p. 249 s.

3 FIORENTIN, Fabio. Attività riparative. In: GIOSTRA, Glauco (a cura di), Carceri: materiali per la riforma. Disponibile in: www.archiviodpc.dirittopenaleuomo.org. Accesso in: 17.6.2015, p. 202.

4 Per un quadro dei principali strumenti della giustizia riparativa, si rinvia ai lavori degli Stati generali dell'esecuzione penale (istituiti con d.m. in data 8 maggio 2015 e articolatisi in diciotto tavoli tematici) e, in particolare, alla relazione conclusiva del Tavolo 13 "Giustizia riparativa, mediazione e tutela delle vittime”, Allegato 3. La relazione è consultabile, insieme a quelle degli altri tavoli e al documento finale redatto dal Comitato di esperti, all'indirizzo https:// www.giustizia.it/giustizia/it/mg_2_19_1_13.page. Accesso in: 5.2.2016.

5 La direttiva 2012/29/UE, che - nel sostituire la decisione quadro 2001/220/ GAI - ha istituito norme minime in materia di diritti, assistenza e protezione delle vittime, definisce così la giustizia riparativa: «qualsiasi procedimento 
ha neppure sfiorato la fase esecutiva ${ }^{6}$. E, da ultimo, è rimasta inattuata se non con riguardo all'ambito minorile ${ }^{7}$ - la legge delega per la riforma dell'ordinamento penitenziario ${ }^{8}$ nella parte in cui poneva le basi di un nuovo corso, sensibile all'esigenza di arricchire, in una prospettiva organica, i contenuti dei percorsi funzionali al recupero sociale del condannato9.

Il quadro, nel complesso, è deludente. Tuttavia, sebbene angusti ${ }^{10}$, non mancano gli spazi per ospitare forme di giustizia riparativa: sono due, per l'espresso riferimento alla vittima, le disposizioni di rilievo ${ }^{11}$.

che permette alla vittima e all'autore del reato di partecipare attivamente, se vi consentono liberamente, alla risoluzione delle questioni risultanti dal reato con l'aiuto di un terzo imparziale» (art. 2.1 lett. $d$ ). Analoga è la definizione che compare al punto 3 Raccomandazione (18) 8 del Comitato dei Ministri del Consiglio d'Europa.

6 V. VICOLI, Daniele. Il ruolo della vittima nella fase esecutiva tra occasioni mancate e scenari futuri. In BARGIS, Marta; BELLUTA, Hervé (a cura di), Vittime di reato e sistema penale. Torino: Giappichelli, 2017, p. 535 s.

7 V. infra, § 6.

8 Art. 1 commi 82 e 85 1. 23 giugno 2017, n. 103. V. GIOSTRA, Glauco; BRONZO, Pasquale (a cura di). Proposte per l'attuazione della delega penitenziaria. Roma: Sapienza Università Editrice, 2017; GIOSTRA, Glauco, La riforma penitenziaria: il lungo e tormentato cammino verso la Costituzione. Diritto penale contemporaneo, 2018, n. 4, p. 119 s.

9 Il riferimento è al criterio direttivo sancito dall'art. 1 comma 85 lett. $f$ l. n. 103 del 2017: «previsione di attività di giustizia riparativa e delle relative procedure, quali momenti qualificanti del percorso di recupero sociale sia in ambito intramurario sia nell'esecuzione delle misure alternative». E', però, caduto nel nulla lo schema di decreto legislativo in materia di giustizia riparativa e mediazione reo-vittima (v. il dossier all'indirizzo https://documenti. camera.it/Leg18/Dossier/Pdf/gi0009.Pdf. Accesso in: 26.6.2018).

10 Alla logica dell' "incontro" con la persona offesa possono essere adattati - pur mancando un esplicito riferimento a quest'ultima - anche i benefici della semilibertà (art. 50 ord. penit.) e della liberazione condizionale (art. 176 c.p.). Il comune denominatore è rappresentato del peso che riveste, ai fini del giudizio sulle possibilità di reinserimento sociale del condannato, la revisione critica del fatto delittuoso: lungo questi binari, può svilupparsi «una ri-lettura del crimine, da parte del reo, che tenga conto anche delle vittime» (MANNOZZI, Grazia. Le aperture alla giustizia riparativa nell'ambito delle misure alternative alla detenzione. Giurisprudenza italiana, 2016, p. 1534).

11 V. KILCHLING Michael; PARLATO, Lucia. Nuove prospettive per la restorative justice in seguito alla direttiva sulla vittima: verso un "diritto alla mediazione”? Germania e Italia a confronto. Cassazione penale, 2015, p. 4197-4198; MANNOZZI, Grazia. Le aperture, cit., p. 1534. 
Una, di centrale importanza sistematica, è quella dell'art. 47 comma 7 ord. penit.: nel verbale descrittivo delle prescrizioni imposte dal giudice nel concedere il beneficio dell'affidamento in prova, deve stabilirsi che il condannato «si adoperi in quanto possibile in favore della vittima del [...] reato» ${ }^{12}$. Lo sfondo è quello del probation penitenziario, le cui origini riposano sulla rinuncia dello Stato all'indefettibilità della pena in vista della rieducazione del reo $^{13}$. E' nel contesto della misura alternativa al carcere che s'innesta un vincolo di solidarietà nei confronti della vittima ${ }^{14}$.

L'altra, di più recente introduzione ${ }^{15}$, s'inserisce nella cornice del lavoro all'esterno: $\mathrm{i}$ detenuti possono essere assegnati a prestare attività a titolo volontario e gratuito in chiave di sostegno alle famiglie delle vittime dei reati commessi (art. 21 comma 4-ter ord. penit.) ${ }^{16}$.

Delineato lo scenario a cui guardare, il nodo da sciogliere è se gli strumenti citati, per fondamento e schema operativo, rispondano ai tratti salienti nella mediazione, declinata nella logica della restorative justice. Per rispondere al quesito, è indispensabile definire taluni principi cardine che, nel tentativo di coniugare l'esecuzione della pena e le pratiche mediative, vanno tenuti a mente.

12 Nel testo originario, la disposizione era meno stringente, poiché configurava come facoltativa la prescrizione in esame, poi divenuta obbligatoria - sia pure con il limite rappresentato dall'inciso «in quanto possibile» - in seguito alle modifiche introdotte dalla 1. 10 ottobre 1986, n. 663.

13 V. FASSONE, Elvio, Voce "Probation e affidamento in prova”. Enciclopedia del diritto. Vol. XXXV. Milano: Giuffrè, 1986, p. 784.

14 E' proprio sull'onda delle prassi originate dalla previsione in esame che il Dipartimento dell'Amministrazione penitenziaria ha deciso, nel 2002, di istituire una Commissione di studio sulla "Mediazione penale e la giustizia riparativa", dai cui lavori sono poi scaturite le "Linee di indirizzo sull'applicazione nell'ambito dell'esecuzione penale dei condannati adulti" (circolare in data 14 giugno 2005): ad essere evidenziati sono aspetti di «indubbia criticità», sulla base, in particolare, degli esiti di due monitoraggi (il secondo relativo all'analisi di 4.511 casi).

15 Ad opera del d.1. $1^{\circ}$ luglio 2013, n. 78, conv. nella 1. 9 agosto 2013, n. 94.

16 V. GIORS, Barbara. Il lavoro all'esterno e gli incentivi volti a favorire l'attività lavorativa dei detenuti. In CAPRIOLI, Francesco; SCOMPARIN, Laura (a cura di), Sovraffollamento carcerario e diritti dei detenuti, Torino: Giappichelli, 2015, p. 146. 


\section{LA MEDIAZIONE NELLA FASE ESECUTIVA: TRATTI DISTINTIVI.}

Brevi riflessioni preliminari sono utili a delimitare il campo d'indagine. In senso stretto, può parlarsi di mediazione in executivis solo dopo l'irrevocabilità della sentenza di condanna ${ }^{17}$. Le dinamiche situate in tale frangente, nel muovere da un definitivo accertamento della colpevolezza, trovano terreno fertile entro il perimetro dei benefici penitenziari e, in specie, delle alternative alla pena detentiva.

Questo dato segna un importante divario rispetto ai percorsi che si esplicano in pendenza della fase di cognizione. L'ipotesi emblematica è quella della sospensione con messa alla prova ${ }^{18}$, la cui fisionomia può risultare funzionale all'obiettivo di ricomporre la frattura che ha origine nel reato ${ }^{19}$. Sul piano teorico, tuttavia, va evidenziato un fattore distintivo:

17 In termini analoghi, v. UBERTIS, Giulio. Relazione. In AA. VV., Accertamento del fatto, alternative al processo, alternative nel processo, Milano: Giuffrè, 2007, p. 148.

18 Nel sistema italiano, tale istituto, inizialmente previsto nel quadro del rito minorile (artt. 28-29 d.p.R. 22 novembre 1988, n. 448), è stato esteso dalla 1. 28 aprile 2014 , n. 67 a quello per adulti (art. 168-bis c.p. s. e art. 464-bis c.p.p. s.). Nei due contesti, la disciplina si caratterizza per un nucleo comune: il giudice può disporre la sospensione del processo e impartire all'imputato delle prescrizioni comportamentali, tra cui quelle dirette a promuovere la mediazione con la persona offesa; ove il percorso trattamentale abbia esito positivo, il reato viene dichiarato estinto con sentenza. Tra le numerose fonti, v. BARTOLI, Laura. La sospensione del procedimento con messa alla prova. Milano: Wolters Kluwer-Cedam, 2020, p. 87 s.; CARACENI, Lina. Voce "Processo penale minorile”. Enciclopedia del diritto. Aggiornamento, v. IV. Milano: Giuffrè, 2000, p. 1037 s.; CESARI, Claudia. Commento all'art. 28. In: GIOSTRA, Glauco (a cura di), Il processo penale minorile, $2^{\mathrm{a}}$ ed., Milano: Giuffrè, 2007, p. 455 s.; COLAMUSSI, Margherita. La messa alla prova. Padova: Cedam, 2010, p. 16 s.; LOGLI, Andrea, La sospensione del processo per messa alla prova tra equivoci dogmatici e limiti operativi. In DANIELE, Marcello; PAULESU, Pier Paolo (a cura di), Strategie di deflazione processuale e rimodulazioni del giudizio in absentia, Torino: Giappichelli, 2016, p. 161; MAFFEO, Vania. I profili processuali della sospensione con messa alla prova. Napoli: Esi, 2017, p. 155 s.; MIRAGLIA, Michela. La messa alla prova dell'imputato adulto. Torino: Giappichelli, 2020, p. 111 s.

19 V., anche per i rilievi critici sulla prevalenza degli aspetti afflittivi rispetto a quelli riparatori, BERTOLINI, Benedetta. La messa alla prova per adulti sotto le lenti della giustizia riparativa. In: MARANDOLA, Antonella; LA REGINA, Katia; APRATI, Roberta (a cura di), Verso un processo penale accelerato, Napoli: Jovene, p. 25 s.; BARTOLI, Laura. La sospensione, cit., p. 184 s.; 
l'eventuale ricorso alle pratiche mediative si colloca in una parentesi dell'iter ordinario, che è suscettibile di riprendere corso ove l'epilogo della prova non sia favorevole ${ }^{20}$.

Ne deriva un diverso inquadramento dei due fenomeni: il probation penitenziario e gli altri benefici hanno come referente una pena inflitta; restano da stabilirne solo le modalità esecutive. I congegni sospensivi, invece, operano in chiave di fuoriuscita dal processo, del quale si auspica ma non è detto che ciò avvenga - la chiusura con una declaratoria di estinzione del reato. Per tale motivo, sebbene caratterizzata da una componente sanzionatoria ${ }^{21}$, la messa alla prova risente, in un panorama segnato da instabili equilibri ${ }^{22}$, della logica sottesa ai riti speciali e non può ascriversi al paradigma dell'esecuzione, con il relativo bagaglio di alternative alla pena detentiva ${ }^{23}$.

COLAMUSSI, Margherita. La messa alla prova, cit., p. 10 s.; MAFFEO, Vania. I profili processuali, cit., p. 116 s.; MIRAGLIA, Michela. La messa alla prova, cit. p. $165 \mathrm{~s}$.

20 V. COLAMUSSI, Margherita. La messa alla prova, cit., p. 217; MIRAGLIA, Michela. La messa alla prova, cit., p. 73.

21 V. BARTOLI, Laura. La sospensione, cit., p. 340 s.; MIRAGLIA, Michela. La messa alla prova, cit., p. 158 s. Tale conclusione, in prima battuta respinta dalla Corte costituzionale (Corte cost., sent. 27 aprile 2018, n. 91), è poi affiorata nelle pronunce che hanno sottolineato le peculiarità della messa alla prova minorile, da ritenersi intrisa di finalità rieducative e quindi, a differenza di quella per adulti, scevra da connotati sanzionatori (Corte cost., sent. 29 marzo 2019, n. 68; sent. 6 luglio 2020, n. 139).

22 Il tema di fondo è quello dei rapporti con la presunzione di non colpevolezza (art. 27 comma 2 Cost.). Nell'escludere l'aggiramento di tale garanzia, Corte cost., sent. 27 aprile 2018, n. 91 ha valorizzato la base consensuale dell'istituto: «il trattamento programmato non è [...] una sanzione penale, eseguibile coattivamente, ma dà luogo a un'attività rimessa alla spontanea osservanza delle prescrizioni da parte dell'imputato, il quale liberamente può farla cessare con l'unica conseguenza che il processo sospeso riprende il suo corso». Tra i numerosi commenti alla pronuncia, v. CESARI, Claudia. La Consulta supera le perplessità e la messa alla prova si radica nel sistema. Giurisprudenza costituzionale, 2018, p. 794 s.; MAFFEO, Vania. Novità sistematiche in tema di messa alla prova. Per una riconsiderazione, costituzionalmente compatibile, del rapporto tra pena e processo. Cassazione penale, 2018, p. 3194 s.; PARLATO, Lucia. La messa alla prova dopo il dictum della Consulta: indenne ma rivisitata e in attesa di nuove censure. Diritto penale contemporaneo, 2019, n. 1, p. $101 \mathrm{~s}$. In tal senso, v. FERRUA, Paolo. Una messa alla prova sul filo del rasoio costituzionale. In: DANIELE, Marcello; PAULESU, Pier Paolo (a cura di), Strategie 


\section{UN QUADRO AMBIVALENTE: VANTAGgI E OSTACOLI.}

Posta questa premessa, va detto che, nella fase dell'esecuzione, si riscontrano indici congeniali all'avvio di un percorso mediativo.

A. Nelle riflessioni dedicate al possibile innesto di simili schemi nel processo penale, uno dei temi centrali riguarda il rischio di attriti e ricadute negative sul versante di irrinunciabili garanzie ${ }^{24}$.

Emerge, in primis, il difficile rapporto con la presunzione d'innocenza (art. 27 comma 2 Cost.). Su questo terreno, la ricerca di un approdo conciliativo genera, se non un'aporia ${ }^{25}$, quanto meno delle tensioni: il pericolo è che, nell'ottica di sanare un conflitto, ne venga obliterata la base logica, vale a dire l'accertamento di responsabilità ${ }^{26}$. Inoltre, vi è l'esigenza di evitare che, nel caso in cui il tentativo non vada a buon fine, le attività compiute influenzino il processo penale. Si pensi, in particolare, a un eventuale uso delle dichiarazioni rese dall'imputato, con l'effetto di eludere il diritto al silenzio e, in termini più generali, scalfire quello di difesa ${ }^{27}$.

di deflazione, cit., p. 184, che sottolinea «il paradosso di una pena 'rieducativa', applicata in pendenza del processo per sfuggire all'effetto 'criminogeno' della pena detentiva».

Il «vizio di origine» della mediazione nell'efficace sintesi di MANNOZZI, Grazia. La giustizia senza spada, Giuffrè, Milano, 2003, p. 240.

Così UBERTIS, Giulio. Relazione, cit., p. 149.

26 V. CESARI, Claudia. Le clausole di irrilevanza del fatto nel sistema processuale penale. Torino: Giappichelli, 2005, p. 102. Secondo l'impostazione seguita da CIAVOLA, Agata. Il contributo della giustizia consensuale e riparativa all'efficienza dei modelli di giurisdizione. Torino: Giappichelli, 2010, p. 256, «si può dire che la responsabilità dell'imputato è oggetto di accertamento, ma cambia la sede: non nel processo, bensì nel corso dell'attività di mediazione». Relativizza il problema ORLANDI, Renzo. La mediazione penale tra finalità riconciliative ed esigenze di giustizia. In: AA. VV., Accertamento del fatto, cit., p. 182, ad avviso del quale «i principi fondamentali, quali, ad esempio, il nemo tenetur se detegere e la presunzione d'innocenza, non irradiano qui loro effetti, giacché chi esperisce il tentativo di mediazione è solitamente disposto a condividere con la controparte una certa versione circa i motivi e le scaturigini del conflitto interpersonale», fermo restando che «l'“episodio" mediazione [...] va poi necessariamente coordinato con il processo penale».

27 Muove da queste premesse l'indirizzo che sostiene l'inutilizzabilità delle dichiarazioni (ORLANDI, Renzo. La mediazione penale, cit., p. 186; PATANÈ, Vania. Ambiti di attuazione di una giustizia conciliativa alternativa a quella 
Ebbene, con il passaggio al contesto dell'esecuzione, i problemi sopra evidenziati svaniscono. Il motivo è molto semplice: come già detto, il tema della colpevolezza risulta "blindato" dalla sentenza irrevocabile. La forza vincolante della presunzione d'innocenza e delle altre garanzie che devono guidare la verifica sulla fondatezza dell'accusa si è ormai esaurita. Non serve, quindi, interrogarsi sui presidi necessari per impedire l'aggiramento di fondamentali principi costituzionali. Da questo punto di vista, il campo si presenta libero da possibili interferenze.

B. Sempre per i risvolti positivi della res iudicata, la strada verso forme di conciliazione è, sul piano operativo, meno irta di ostacoli.

Si ritiene che, per il lieto fine del dialogo tra le parti antagoniste, siano indispensabili alcune condizioni. Tra queste, rileva in modo significativo la disponibilità dell'autore del reato a riconoscere la propria colpa: se non nei termini di una confessione, almeno ammettendo il nucleo fondante dell'illecito ${ }^{28}$. Inoltre, in un'ottica che abbracci anche la vittima, il compito del mediatore è agevolato ove sui fatti principali della vicenda vi sia una convergenza di vedute ${ }^{29}$.

Se questi sono i capisaldi di un confronto funzionale a ricucire la trama dei rapporti interpersonali, non vi è dubbio che la fase dell'esecuzione offra notevoli margini di manovra. Di fronte a una sentenza definitiva, crescono le chanches che il condannato, nell'intraprendere un percorso di responsabilizzazione, riconosca l'offesa arrecata e la necessità di ripararla.

penale: la mediazione. In: MESTITZ, Anna (a cura di), Mediazione penale: chi, dove, come e quando, Roma: Carrocci editore, 2004, p. 29). Per alcuni rilievi critici sull'adeguatezza di tale rimedio, v., con specifico riguardo al tentativo di conciliazione previsto nell'ambito del procedimento davanti al giudice di pace (art. 29 d. lgs. 28 agosto 2000, n. 274), CESARI, Claudia. Le clausole di irrilevanza, cit., p. 93.

28 V. CERETTI, Adolfo. Mediazione penale e giustizia. In: AA. VV., La mediazione penale in ambito minorile: applicazioni e prospettive, Milano: Franco Angeli, 1999, p. 99. V. anche PATANẼ, Vania. La mediazione. In: GIOSTRA, Glauco; ILLUMINATI, Giulio (a cura di), Il giudice di pace nella giurisdizione penale, Torino: Giappichelli, 2000, p. 365. Insistono su questo profilo anche l'art. 12.1 lett. $c$ direttiva 2012/29/UE (v. KILCHLING Michael; PARLATO, Lucia. Nuove prospettive, cit., p. 4192) e il punto 30 Raccomandazione (18) 8.

29 CIAVOLA, Agata. Il contributo della giustizia consensuale, cit., p. 261, secondo la quale «senza [tale] intesa la possibilità di raggiungere un accordo risulta limitata, se non esclusa». 
Al tempo stesso, lo scambio comunicativo è favorito dalla ricostruzione storica che l'accertamento giudiziale ha cristallizzato. Tale circostanza giova al mediatore, che può far leva su solide basi di partenza per muovere alla ricerca di una soluzione del conflitto.

Infine, una volta chiusa la fase cognitiva del processo, diventa naturale valorizzare le componenti personologiche dell'episodio criminoso, in modo da porre l'accento sul «reato come vicenda personale, fatto di una comunicazione interrotta, di legami sociali infranti, di conflittualità psicologiche articolate e di ragioni profonde $»^{30}$.

Agli innegabili vantaggi sopra evidenziati fanno, però, da contraltare alcuni nodi problematici, che rendono difficile il connubio tra la sfera esecutiva e la mediazione.

A. Lo scarto temporale che separa il reato e l'entrata in scena del mediatore nuoce all'approccio di tipo conciliativo. Di frequente, può verificarsi che la situazione conflittuale risulti «ormai "incancrenita" ${ }^{31}$; nel ricomporla, è quindi inevitabile andare incontro a maggiori resistenze ${ }^{32}$ : un dato che fa scemare le possibilità di successo ${ }^{33}$. Trascorsi molti anni dal fatto, inoltre, è più probabile che la vittima, nel superare il trauma patito, abbia raggiunto un equilibrio esistenziale; in simili casi, dal tentativo di mediazione potrebbe scaturire una nuova violenza ${ }^{34}$.

30 CESARI, Claudia. Le clausole di irrilevanza, cit., p. 93, la quale osserva come indagini di questo tipo, proprio perché rischiano di sviare l'accertamento di merito, siano «solitamente riservate alle verifiche della fase esecutiva».

31 DELLA CASA, Franco. Affidamento al servizio sociale o (pura e semplice) "pay-back sanction"? Equivoci sul significato dell'art. 47 co. 7 o.p. La legislazione penale, 2004, p. 382.

32 V. MANNOZZI, Grazia; LODIGIANI, Giovanni Angelo. La giustizia riparativa, cit., p. 252.

33 In questo senso, le citate linee di indirizzo (v. supra, nota 14) individuano l'arco temporale massimo tra il reato e l'inizio dell'opera di mediazione in cinque anni, a fronte dei dieci spesso registrati in sede di monitoraggio. In dottrina, v. DI CHIARA, Giuseppe. La premura e la clessidra: i tempi della mediazione penale. Diritto penale e processo, 2015, p. 381, il quale, nel distinguere «il tempo intercorso tra l'epoca del commesso reato e l'avvio dell'attività di mediazione» dal «fluire del tempo entro la conca della mediazione», osserva come il primo vada dosato in modo da risultare non troppo breve né troppo ampio.

34 Ne deriva la necessità, come sottolinea anche la Raccomandazione (18) 8 (punto 36 s.), di costruire un percorso normativo, deontologico e 
Il rilievo offre lo spunto per una riflessione di più ampio respiro. Sul piano delle opzioni di sistema, la scelta di relegare al solo momento esecutivo le pratiche mediative sarebbe senz'altro miope e, nel complesso, non potrebbe che avere un impatto modesto. Se il disegno è quello d'incentivare gli istituti a sfondo riparativo, l'esecuzione della pena deve essere l'ultimo frangente utile, nell'ottica - cioè - di offrire un'opportunità ulteriore rispetto a percorsi che si sviluppino entro le coordinate della fase cognitiva ${ }^{35}$. In caso contrario, nel quadro d'insieme, il ruolo della mediazione apparirebbe fortemente svilito.

B. Requisito indefettibile delle forme di giustizia conciliativa è il consenso delle parti, da intendersi quale assenza di pressioni e spinte coercitive $^{36}$. Per l'autore del reato, tale necessaria spontaneità può risentire, fino ad essere alterata, dell'influenza che la condanna ormai da espiare esercita. Negli stadi anteriori, la pena resta una minaccia, sebbene - di pari passo all'evolversi del procedimento - sempre più intensa; nelle dinamiche dell'esecuzione, diventa una realtà che incombe sul condannato. Non è facile stabilire in quale grado il dato possa incidere sulla libera adesione al dialogo con la persona offesa. Sembra, tuttavia, evidente il pericolo di vedere snaturata l'essenza dello schema riparativo ${ }^{37}$, anche a causa della

metodologico che delinei le modalità di approccio alla vittima del reato (v. MANNOZZI, Grazia. Le aperture, cit., p. 1534). In termini più generali, sul tema della c.d. vittimizzazione secondaria, v. ALLEGREZZA, Silvia. La riscoperta della vittima nella giustizia penale europea. In: AA. VV., Lo scudo e la spada, Torino: Giappichelli, 2012, p. 17 s.

35 E' in sintonia con questa logica l'ampiezza operativa che traspare dal punto 6 Raccomandazione (18) 8: «La giustizia riparativa può essere utilizzata in ogni fase del procedimento penale. Ad esempio, può essere associata a una forma di diversione dall'arresto, dall'esercizio dell'azione penale o dal perseguimento penale del fatto, può essere usata congiuntamente all'archiviazione di polizia o giudiziaria, può intervenire prima o parallelamente al perseguimento penale del fatto, può collocarsi tra l'accertamento di responsabilità e la comminazione di una pena, può costituire parte di una pena o intervenire dopo la comminazione o l'espiazione della stessa».

36 V. CIAVOLA, Agata. Il contributo della giustizia consensuale, cit., p. 258; PATANÈ, Vania. Ambiti di attuazione, cit., p. 24. Le Raccomandazioni (99) 19 e (18) 8 nonché la direttiva 2012/29/UE sono categoriche nel fare riferimento all'esigenza di un libero consenso delle parti.

37 Osserva CIARDIELLO, Patrizia. Riparazione e mediazione nell'ambito dell'esecuzione penale per adulti. Rassegna penitenziaria e criminologica, 2007, $\mathrm{n}$. 
posizione di forza in cui viene a trovarsi la vittima: si pensi agli effetti distorsivi che l'impulso a evitare la pena detentiva potrebbe avere sul quantum di un eventuale ristoro economico.

Poche battute sono sufficienti per tirare le somme dell'analisi svolta. Nel quadro dei rapporti tra l'esecuzione della pena e i meccanismi della restorative justice si colgono luci e ombre. A risultare ambivalente è un tratto di fondo: l'irrevocabilità della sentenza di condanna. E' intorno al carattere stabile dell'accertamento che ruotano quelle circostanze capaci di agevolare un esito conciliativo; la mediazione, però, oltre a rivelarsi più ostica in quanto sul piano temporale lontana dall'offesa, risente degli effetti sanzionatori che la res iudicata implica.

\section{IL NESSO CON IL FINALISMO RIEDUCATIVO DELLA PENA.}

Delineato lo scenario, è fondamentale interrogarsi sulla ratio che, nella fase esecutiva, deve animare gli strumenti normativi ispirati al paradigma della mediazione.

Anche su questo versante, emergono significative differenze rispetto agli istituti che operano entro l'area del processo di cognizione. Per questi ultimi, sebbene il panorama si presenti composito, una nota distintiva può cogliersi nelle finalità di economia processuale: se $\mathrm{i}$ meccanismi mutano, lo sbocco auspicato resta identico, vale a dire la fine anticipata della vicenda giudiziaria. Pertanto, la ricorrente chiave di lettura, pur non immune da rilievi critici ${ }^{38}$, tende a collocare le pratiche mediative nel solco della diversion ${ }^{39}$.

2, p. 98, come «il rischio di strumentalizzazione è intrinseco al sistema premiale, fondato sullo scambio penitenziario: l'ammissione ai benefici [...] va [...] conquistata dal condannato dimostrando la propria "meritevolezza" ed affidabilità personale e sociale».

38 V. ORLANDI, Renzo. La mediazione penale, cit., p. 186.

39 UBERTIS, Giulio. Relazione, cit., p. 147. Nell'ordinamento italiano, il quadro si presenta eclettico in ragione della specificità che caratterizza i corpi normativi extra codicem: il rito minorile e quello davanti al giudice di pace. La spinta ad aprire, nelle maglie del processo, canali comunicativi che coinvolgano l'autore del reato e la persona offesa riflette la tensione ideale di tali microsistemi (CESARI, Claudia. Efficienza della giurisdizione penale e strategie di depenalizzazione processuale. In: KOSTORIS, Roberto E. (a cura di), 
Per evidenti motivi, tale imprinting è destinato a svanire nello stadio di esecuzione della pena; di riflesso, vanno ricercati altrove gli scopi sottesi ai percorsi dalla potenziale valenza mediativa.

Invero, politiche d'indole pragmatica potrebbero tornare in gioco nell'ambito dei rimedi a quel ciclico e irrisolto male che affligge il sistema penitenziario italiano: il sovraffollamento ${ }^{40}$. In tal senso, l'ottica - come nel caso dell'art. 47 comma 7 ord. pen. - sarebbe quella d'incentivare il ricorso alla restorative justice per favorire l'accesso alle misure alternative al carcere; ne deriverebbero, infatti, vantaggi in termini di decremento della popolazione detenuta. Tale impostazione è da rifiutare, in quanto troppo angusta e incapace di fornire solide basi concettuali alle pratiche mediative. Non si nega che queste ultime possano avere ricadute apprezzabili sul piano della deflazione carceraria; si tratta, però, di effetti secondari, da ritenersi estranei al fondamento teleologico delle stesse.

La tensione a riparare il danno derivante dal reato e, più in generale, a intraprendere un cammino riconciliativo deve inserirsi in un programma che miri al reinserimento sociale del condannato ${ }^{41}$. La cornice entro la quale sviluppare lo schema della mediazione è quella rieducativa,

La ragionevole durata del processo, Torino: Giappichelli, 2005, p. 95-96). In particolare, la ricerca del dialogo e l'importanza delle condotte riparatorie sono valori in linea con l'opzione per un diritto penale "mite", soprattutto là dove ad assumere un ruolo centrale sono le esigenze rieducative e assistenziali dell'imputato minorenne.

40 Al 31.5.2021, a fronte di una capienza regolamentare di 50.780 posti, risultano detenute (a titolo definitivo o cautelare) 53.660 persone (Ministero della giustizia, Dipartimento dell'amministrazione penitenziaria: https: // www.giustizia.it/giustizia/it/mg_1_14_1.page?contentId=SST334403\&previsiousPage=mg_1_14. Accesso in: 21.6.2021). Se rispetto al 29.2.2020 vi è stata una diminuzione - dovuta alle misure adottate per fronteggiare l'emergenza sanitaria da COVID-19 - di circa 7.600 unità, si è poi registrata un'inversione di tendenza (al 31.12.2020 il numero delle persone detenute era di 53.364). V. Associazione Antigone, Oltre il virus - XVII rapporto sulle condizioni di detenzione, consultabile all'indirizzo https: //www.rapportoantigone.it/diciassettesimo-rapporto-sulle-condizioni-di-detenzione/. Accesso in: 21.6.2021.

41 FIORENTIN, Fabio. Riparazione e mediazione dopo il giudizio nel quadro dell'esecuzione penitenziaria e delle misure alternative alla detenzione. $L a$ legislazione penale, 2004, p. 394 e 397. 
segnata dallo stimolo affinché il soggetto rivisiti in chiave critica l'illecito commesso $^{42}$ e torni ad aderire alle regole della convivenza civile ${ }^{43}$.

In questa concezione progettuale della pena ${ }^{44}$ emerge la centralità della vittima ${ }^{45}$. Nella fase cognitiva, a catalizzare gli interessi rivali sono l'accertamento della responsabilità e la commisurazione della pena. L'esperienza del processo finisce per "separare" autore del reato e persona offesa: a tale logica la giustizia riparativa ne sostituisce una opposta, che guarda all'“inclusione”. Il fulcro sta nel soddisfare, attraverso la tessitura di rapporti intersoggettivi, la domanda di riconoscimento che proviene dalle parti. Pertanto, assumono un rilievo preminente il vissuto criminale e quello di vittimizzazione ${ }^{46}$ : affiorano così fatti nuovi, rimasti

42 A questa prospettiva si salda l'unico appiglio normativo che permette di coinvolgere la vittima nelle logiche trattamentali: in base all'art. 27 comma 1 d.p.R. 30 giugno 2000, n. 230 («Regolamento recante norme sull'ordinamento penitenziario e sulle misure privative e limitative della libertà personale»), nel corso delle attività d'osservazione intramuraria, «va espletata, con il consenso del condannato [...], una riflessione sulle condotte antigiuridiche poste in essere, sulle motivazioni e sulle conseguenze negative delle stesse per l'interessato medesimo e sulle possibili azioni di riparazione delle conseguenze del reato, incluso il risarcimento dovuto alla persona offesa». A tale previsione si affianca, per il trattamento in ambiente esterno, quella dell'art. 118 comma 8 lett. $d$ d.p.R. 30 giugno 2000, n. 230: gli interventi degli uffici di esecuzione penale esterna (U.E.P.E.) devono caratterizzarsi per la «sollecitazione a una valutazione critica adeguata, da parte della persona, degli atteggiamenti che sono stati alla base della condotta penalmente sanzionata, nella prospettiva di un reinserimento compiuto e duraturo».

43 SCOMPARIN, Laura. Quale giustizia riparativa dopo la conclusione del processo? La legislazione penale, 2004, p. 407-408.

44 L'idea è quella del passaggio da una «pena subita» ad una «pena agìta», cioè una «sanzione che rimane sì negativa, privativa o limitativa di diritti, ma al contempo implica un comportamento attivo del soggetto» (DONINI, Massimo. Il delitto riparato. Una disequazione che può trasformare il sistema sanzionatorio. Diritto penale contemporaneo, Riv. trim., 2015, n. 2, p. 241).

In tal senso, nel testo, si ripercorre il pensiero di MANNOZZI, Grazia. La reintegrazione sociale del condannato tra rieducazione, riparazione ed empatia. Diritto penale e processo, 2012, p. 849. Al tempo stesso, va chiarito che una rinnovata sensibilità per il ruolo della vittima non potrebbe tradursi in poteri di veto sul percorso trattamentale e rieducativo del condannato; sullo specifico tema dei rapporti tra accesso alle misure alternative e obblighi risarcitori, v. infra, § 5 .

46 «Se, per la vittima, la comunicazione del vissuto risponde ad una esigenza di "riconoscimento", il racconto dell'esperienza criminale costituisce, per il 
fuori o ai margini del fenomeno processuale. Questa strada conduce a individualizzare non solo il trattamento del reo, ma anche quello della persona offesa ${ }^{47}$.

Proprio nell'ottica appena considerata, bisogna chiedersi se e, nel caso, in quali termini la tipologia del reato debba incidere sulla sfera di operatività che, in executivis, è opportuno assegnare alla mediazione.

L'interrogativo presenta molteplici sfumature, legate alle scelte di fondo a cui ispirare la geometria delle relazioni tra condannato e vittima. In termini generali, va sposata la linea secondo cui «non si ravvisano gli estremi per la compilazione di un elenco di reati o di condannati» da escludere a priori ${ }^{48}$. A rilevare non sono la pena edittale o il nomen juris del reato, ma le concrete circostanze dell'episodio criminoso $^{49}$ : è da queste ultime che dipendono il conflitto interpersonale e, quindi, «la realistica possibilità di un suo superamento attraverso adeguate "terapie comunicative" $\gg^{50}$. Del resto, sono in special modo i delitti che minano le radici di una comunità a mettere in luce la «valenza profonda» della giustizia riparativa ${ }^{51}$.

reo, un tassello fondamentale per lavorare sul senso di colpa e perciò, in definitiva, su quei fattori criminogenetici connessi al ricorso alle c.d. "tecniche di neutralizzazione" che, se non affrontati, facilitano la ricaduta nel reato» (MANNOZZI, Grazia. La reintegrazione sociale, cit., p. 849).

47 DONINI, Massimo. Il delitto riparato, cit., p. 244; MANNOZZI, Grazia. Le aperture, cit., p. 1533. In tal senso, s'impone un notevole salto di qualità: ad «essere culturalmente distante dal nostro ordinamento è la stessa idea di una presa in carico della vittima» (CATALANO, Elena Maria. La tutela della vittima nella direttiva 2012/29/UE e nella giurisprudenza delle corti europee. Rivista italiana di diritto e procedura penale, 2014, p. 1805).

DELLA CASA, Franco. Affidamento al servizio sociale, cit., p. 384.

DELLA CASA, Franco. Affidamento al servizio sociale, cit., p. 384. Diversa è l'opinione di PATANÈ, Vania. Ambiti di attuazione, cit., p. 23, che suggerisce di mettere al bando «quelle tipologie di reato espressive di forme di conflittualità particolarmente gravi, ontologicamente inconciliabili con qualsiasi forma di negoziazione». ORLANDI, Renzo. La mediazione penale, cit., p. 177.

51 MANNOZZI, Grazia. La reintegrazione sociale, cit., p. 850. Il dato teorico trova riscontro nei risultati empirici: alla maggiore gravità dei reati corrispondono più significativi effetti di contenimento della recidiva, che arrivano a svanire nel caso dei c.d. illeciti senza vittima (MANNOZZI, Grazia; LODIGIANI, Giovanni Angelo. La giustizia riparativa, cit., p. 365). Sul piano sociologico, 


\section{UN PANORAMA APPLICATIVO DELUDENTE.}

Da un autentico modello di restorative justice l'attuale realtà applicativa è, con rare eccezioni ${ }^{52}$, molto lontana.

In prima battuta, è sin troppo facile rimarcare la mancanza di una figura che possa agire nella veste di arbitro. Non bisogna cadere per evidenti motivi - nell'equivoco di elevare il giudice a protagonista dei rapporti tra il reo e la vittima ${ }^{53}$; e nemmeno può farsi leva, in quanto soggetti sforniti della necessaria imparzialità e formazione ${ }^{54}$, sugli operatori penitenziari ${ }^{55}$. Poco consono è anche il ruolo degli addetti agli uffici locali di esecuzione penale esterna (U.E.P.E.): le funzioni istituzionali (art. 72 ord. penit.) non favoriscono un approccio neutrale ${ }^{56}$.

In linea più generale, nell'esperienza italiana, il modello del probation si è rivelato inidoneo a recepire input di natura riparativa. A pesare è stata la crisi d'identità che ha caratterizzato la storia dell'affidamento in prova. Tale misura, nel disegno originario, mirava a soddisfare obiettivi di riabilitazione del condannato: sulla base di questo scopo

di particolare impatto è l'esperienza narrata da BERTAGNA, Guido; CERETTI, Adolfo; MAZZUCCATO, Chiara. Il libro dell'incontro. Milano: Il Saggiatore, 2015: un percorso iniziato nel 2007 e sviluppatosi in un arco temporale di sette anni segnati da incontri, in situazioni di vita comune, fra persone coinvolte nella c.d. lotta armata degli anni '70 e vittime o familiari di vittime dei delitti di quel periodo storico.

52 Un caso virtuoso è quello deciso dal Tribunale di sorveglianza di Venezia (l'ordinanza è pubblicata in Diritto penale e processo, 2012, p. 833): nell'ammettere alla semilibertà un condannato alla pena dell'ergastolo, si è tenuto conto anche di un progressivo riavvicinamento alle vittime dei reati grazie a una forma di mediazione "surrogata", cioè con la vittima di reati analoghi a quelli commessi.

53 Lo sottolinea SCOMPARIN, Laura. Quale giustizia riparativa, cit., p. 413.

54 E significativo che il considerando 63 della direttiva 29/2012/UE insista sulla affidabilità dei servizi di assistenza, che devono essere in grado di fornire alle vittime un sostegno «rispettoso, sensibile, professionale e non discriminatorio».

55 Lo rileva BERTAGNINI, Elisa. Mediazione penale, giustizia riparativa e lavoro gratuito alla luce di qualche esperienza pratica. La legislazione penale, 2004, p. 404.

56 V., con riguardo alla figura degli assistenti sociali in servizio presso gli U.E.P.E. (art. 81 ord. penit.), BERTAGNINI, Elisa. Mediazione penale, cit., p. 402. 
era ritagliata la cerchia dei destinatari, vale a dire persone socialmente disagiate, responsabili di illeciti non gravi ${ }^{57}$. Nel corso del tempo, questa idea finalistica è stata erosa. Due i fattori, tra loro sinergici: le carenze strutturali del sistema, incapace di attuare il paradigma dell'art. 27 comma 3 Cost.; il trend legislativo nel senso di estendere la sfera applicativa dell'affidamento in prova ${ }^{58}$.

E' a quest'ultimo profilo che si salda la "scoperta" delle prescrizioni ex art. 47 comma 7 ord. penit. Alla fine degli anni novanta, al vaglio dei tribunali di sorveglianza sono giunti casi dai tratti peculiari ${ }^{59}$ : la particolare rilevanza dell'offesa cagionata dal reato (in specie, delitti contro la pubblica amministrazione e il patrimonio); lo status dei colpevoli, fin troppo integrati nel tessuto sociale e, di certo, non bisognosi di interventi riabilitativi ${ }^{60}$. In tali fattispecie, era inevitabile che l'affidamento in prova, la cui tensione rieducativa risultava già svilita, si rivelasse «una scatola vuota, priva di qualsiasi contenuto risocializzante» ${ }^{61}$. Così, in simile scenario, si è affermato l'indirizzo incline a rendere più pregnante la misura alternativa attraverso obblighi che, in nome di asserite istanze riparative, imponessero forme di risarcimento della vittima ovvero lavori di pubblica utilità ${ }^{62}$. Alla base vi era la tesi - sostenuta da alcuni tribunali di sorveglianza - secondo cui le prescrizioni dell'art. 47 comma 7 ord. penit. potrebbero avere quale beneficiaria anche la collettività ${ }^{3}$. A frenare simili opzioni è poi stata la giurisprudenza di legittimità, che ne ha messo in luce l'errore di fondo: l'impronta esclusivamente afflittiva, tale da

57 V. CARNEVALE, Stefania. Accesso alle misure alternative. In: GIOSTRA, Glauco (a cura di), Carceri: materiali per la riforma, cit., p. 109.

58 Ripercorre i passaggi di tale evoluzione DELLA CASA, Franco. Misure alternative ed effettività della pena tra realtà e prospettive., Giustizia penale, 2001, II, c. $69 \mathrm{~s}$.

59 Per la rassegna di qelli più significativi, v. FIORENTIN, Fabio. Riparazione e mediazione, cit., p. 390-391.

60 V. DELLA CASA, Franco. Affidamento al servizio sociale, cit., p. 381.

61 MONTEVERDE, Lino. Mediazione e riparazione dopo il giudizio: l'esperienza della magistratura di sorveglianza. Minorigiustizia, 1999, n. 2, p. 87.

62 V. MONTEVERDE, Lino. Mediazione e riparazione, cit., p. 88.

63 V. MONTEVERDE, Lino. Mediazione e riparazione, cit., p. 87-88. 
tradursi in un carico sanzionatorio supplementare, non giustificato dalla condotta criminosa ${ }^{64}$.

Sul punto, per evitare che la giustizia riparativa diventi la «maschera» ${ }^{65}$ di un approccio solo in apparenza ispirato al modello rieducativo, è bene fare chiarezza. Non va escluso che la restorative justice possa caratterizzarsi per una dimensione collettiva ${ }^{66}$. E' indispensabile, però, che si esplichi la logica del dialogo ${ }^{67}$ : la comunità non può essere mera destinataria di una riparazione; deve anche partecipare nella veste di «attore sociale» al percorso di riconciliazione ${ }^{68}$.

In tal senso, apre uno spiraglio la recente previsione dei progetti di pubblica utilità nel cui ambito i detenuti possono chiedere di lavorare a titolo gratuito (art. 20-ter comma 1 ord. penit. ${ }^{69}$ ). Tale attività è annoverata

64 Cass., sez. I, 23 novembre 2001, n. 410, in C.E.D., n. 220439.

65 In questi termini, PARISI, Francesco. La restorative justice alla ricerca di identità e legittimazione. Disponibile in: www.archiviodpc.dirittopenaleuomo.org. Accesso in: 24.12.2014, p. 6. In senso più ampio, v. le osservazioni critiche di DELLA CASA, Franco. Misure alternative, cit., c. 71, che all'origine delle tendenze in esame, individua la colpevole inerzia del legislatore nel forgiare una misura - analoga al community service order o al travail d'interét générale di Paesi stranieri - «adatta ai condannati rispetto ai quali sia da escludere un retroterra di ipo-integrazione sociale».

66 Sul tema, mentre la direttiva 2012/29/UE risente di una visione "tradizionale" (la vittima, ai sensi dell'art. 2.1 lett. $a$, è «una persona fisica che ha subito un danno, anche fisico, mentale o emotivo, o perdite economiche che sono stati causati direttamente da un reato»), la Raccomandazione (18) 8 si caratterizza per esplicite aperture: il pregiudizio da riparare può riguardare la società (punto 13); tra gli interventi a carattere innovativo, sono annoverati i programmi riparativi di comunità (punto 59). Sulle c.d. vittime collettive, v. ALLEGREZZA, Silvia. La riscoperta della vittima, cit., p. 13. Per un quadro delle fonti sovranazionali che contemplano una definizione di vittima, v. BELLUTA, Hervé. Quale ruolo per la vittima nel processo penale italiano? Revista Brasileira de Direito Processual Penal, 2019, n. 1, p. 77 s.

Lo strumento operativo è quello denominato community group conferencing: v. la relazione conclusiva del Tavolo 13 "Giustizia riparativa, mediazione e tutela delle vittime”, Allegato 3.

68 Relazione conclusiva del Tavolo 13 "Giustizia riparativa, mediazione e tutela delle vittime", Allegato 3.

69 Inserito dal d.lgs. 2 ottobre 2018, n. 124. In base al comma 2, beneficiari dell'attività lavorativa possono essere amministrazioni dello Stato, regioni, province, comuni, comunità montane, unioni di comuni, aziende sanitarie locali, enti o organizzazioni di assistenza sociale, sanitaria e di volontariato. 
tra gli elementi fondamentali del trattamento rieducativo (art. 15 ord. penit.) e deve svolgersi in modo idoneo a garantirne l'attuazione (art. 20 -ter comma 6 ord. penit.). Alla luce di questo nesso, fulcro del public work penitenziario diventa l' "impegnarsi per gli altri", che si traduce in un contributo al «progresso materiale o spirituale della società» (art. 4 comma 2 Cost. $)^{70}$. Sebbene manchi un espresso richiamo alla giustizia riparativa, l'impostazione di fondo si presta, in linea teorica, a recepirne i dettami.

In tema di lavoro, nessun passo in avanti si registra, invece, sul versante dei conflitti di origine intersoggettiva. L'art. 21 comma 4-ter ord. penit., la cui applicazione è risultata molto circoscritta ${ }^{71}$, consente - come detto - l'impiego dei detenuti, in forma volontaria e gratuita, a sostegno delle famiglie vittime dei reati da loro commessi. Tale risorsa patisce un ostacolo che è difficile da superare: lo schema incentrato sul lavoro relega ai margini la componente comunicativo-relazionale ${ }^{72}$. Nell'ottica di estendere la mediazione al contesto familiare, la strada maestra è un'altra: il ricorso al c.d. family group conferencing, modalità che rimane fedele al criterio metodologico dell'“incontro"73.

Il disorientamento concettuale si rinviene, inoltre, nella tendenza a sovrapporre due piani distinti: le iniziative riparatorie e il risarcimento del danno. Le prime hanno una valenza molto più ampia e abbracciano svariate forme; non possono, dunque, ridursi alla monetizzazione dell'offesa subita dalla vittima, sulla falsariga degli obblighi sanciti dall'art. 185 c.p. ${ }^{74}$. Sul punto, non aiutano a mettere

70 V. BRONZO, Pasquale. Il lavoro come fulcro del trattamento rieducativo. In: BRONZO, Pasquale; SIRACUSANO, Fabrizio; VICOLI, Daniele (a cura di), La riforma penitenziaria: novità e omissioni del nuovo "garantismo carcerario", Torino: Giappichelli, 2019, p. 153.

71 V. COMMISSIONE GIOSTRA. Il Progetto di riforma penitenziaria, Roma: Nuova Editrice Universitaria, 2019, p. 161.

72 V. MANNOZZI, Grazia. Le aperture, cit., p. 1534, che auspica l'abrogazione o, comunque, la revisione dell'art. 21 comma 4-ter ord. penit.

73 Sul dialogo allargato ai gruppi parentali, v. MANNOZZI, Grazia; LODIGIANI, Giovanni Angelo. La giustizia riparativa, cit., p. 274 s.

74 Va detto che a generare una certa confusione è anche il citato (v. supra, nota 42) art. 27 comma 1 d.p.R. n. 230 del 2000: v. CIARDIELLO, Patrizia. Riparazione e mediazione, cit., p. 100. Nell'ottica di ribadire il discrimine, è significativo che, nella disciplina di alcuni benefici penitenziari, figuri un espresso 
ordine le oscillazioni della giurisprudenza circa il peso da attribuire al ristoro del danno nell'economia dello scrutinio sulla concedibilità della misura alternativa. Non mancano, infatti, pronunce secondo le quali «l'ingiustificata indisponibilità del condannato a risarcire la vittima [...] rientra pur sempre tra gli elementi di segno negativo valutabili per il diniego» del beneficio ex art. 47 ord. penit. ${ }^{75}$.

\section{Conclusioni.}

Il panorama complessivo denota l'assenza dei requisiti minimi indispendabili per declinare, in modo genuino, l'archetipo della riparazione. Sulla base di una palese confusione nominalistica, sono ascritti al modello della restorative justice "impegni” che, insensibili allla forza empatica del dialogo, assumono la fisionomia di un dovere, il cui adempimento serve al reo per usufruire di un premio. Di pari passo, la vittima finisce per essere svilita e divenire il veicolo di logiche punitive.

Un cambio di rotta si deve al d.lgs. 2 ottobre 2018, n. 121, che ha varato la riforma dell'ordinamento penitenziario minorile: l'art. 1 comma 2 assegna all'esecuzione penale il compito di «favorire percorsi

richiamo all'adempimento delle obbligazioni civili derivanti dal reato: è il caso della liberazione condizionale (art. 176 comma 4 c.p.) e della riabilitazione (art. 179 comma 6 n. 2 c.p.).

75 Cass., sez. I, 25 settembre 2007, n. 39474, Arnesano. Cassazione penale, 2009, p. 1199, che segue l'orientamento già espresso da Cass., sez. I, 9 luglio 2001, n. 30785, Iegiani, in C.E.D., n. 219606. Secondo l'indirizzo maggioritario, il risarcimento del danno rileva non quale prerequisito per accedere alla misura alternativa, ma sul piano del giudizio prognostico circa l'idoneità della stessa a soddisfare gli scopi rieducativi; di conseguenza, è nell'ambito di tale valutazione che entrano in gioco le prescrizioni ex art. 47 comma 7 ord. penit., il cui rispetto è poi destinato a incidere sull'esito della prova (v., tra le altre, Cass., sez. I, 17 novembre 2009, n. 47126, Colatore, in C.E.D., n. 245886; Cass., sez. I, 19 maggio 2009, n. 23047, Avanzi, in C.E.D., n. 244070; Cass., sez. I, 27 maggio 2004, n. 37049, Zampolini, in C.E.D., n. 230361; Cass., sez. I, 8 marzo 2001, n. 15098, Gammaidoni, in C.E.D., 218405). Al riguardo, anche per ulteriori riferimenti giurisprudenziali, v. FIORENTIN, Fabio. Uscito dalla porta, rientra dalla finestra l'obbligo del risarcimento del danno per la concessione dell'affidamento in prova del servizio sociale? Cassazione penale, 2009, p. 1200 s. 
di giustizia riparativa e di mediazione con le vittime di reato ${ }^{76}$. La centralità sistematica della previsione trova riscontro nell'ampiezza dei confini applicativi, che spaziano dal trattamento intramurario alle misure alternative al carcere. Ne deriva che sono plurime le tecniche suscettibili di essere sfruttate; è così possibile calibrare l'intervento sulla specifica vicenda, la cui gravità - derivante dal titolo di reato o dalla pena inflitta - non comporta aprioristici limiti. Il punto debole, però, sta nella mancata disciplina delle procedure e delle regole da osservare: tale vuoto, che sottende una delega "in bianco" agli operatori, rischia di mettere un freno all'esplicarsi del paradigma riparativo e, forse, rivela il persistente scetticismo del legislatore. In ogni caso, è da rimarcare la scelta di valore, che ha un significato fortemente innovativo: l'aupicio è che nell'ambito minorile possano attecchire un diverso modello di esecuzione e prassi virtuose, poi da estendere - come avvenuto in altre occasioni - all'universo degli adulti ${ }^{77}$.

\section{Bibliografia}

ALLEGREZZA, Silvia. La riscoperta della vittima nella giustizia penale europea. In: AA. VV., Lo scudo e la spada, Torino: Giappichelli, 2012, p. 1.

76 V. CESARI, Claudia. La giustizia riparativa nel sistema penitenziario minorile: un nuovo orizzonte ancora incerto. In: CARACENI, Lina; COPPETTA Maria Grazia (a cura di), L'esecuzione delle pene nei confronti dei minorenni, Torino: Giappichelli, 2019, p. 47 s.

77 A questo riguardo sono da menzionare le proposte di emendamenti al disegno di legge A.C. 2435 («Delega al Governo per l'efficienza del processo penale e disposizioni per una celere definizione dei procedimenti giudiziari pendenti presso le corti d'appello») formulate dalla Commissione di studio nominata dal Ministro della giustizia con decreto del 16 marzo 2021 (il testo della relazione è consultabile all'indirizzo https://www.sistemapenale.it/it/documenti/relazione-commissione-lattanzi-riforma-giustizia-penale. Accesso in: 16.7.2021). In particolare, l'art. 9-quinquies contempla la delega a varare una disciplina organica della giustizia riparativa («quanto a nozione, principali programmi, garanzie, persone legittimate a partecipare») sulla base di puntuali criteri direttivi, con possibilità di usufruire dei relativi percorsi «senza preclusioni in relazione alla gravità dei reati» e in un'ottica di sistema («in ogni stato e grado del procedimento di merito, nell'ambito degli istituti previsti dal codice penale, dal codice di procedura penale, dall'ordinamento penitenziario, dall'ordinamento minorile e da leggi speciali»). 
BARTOLI, Laura. La sospensione del procedimento con messa alla prova. Milano: Wolters Kluwer-Cedam, 2020.

BELLUTA, Hervé. Quale ruolo per la vittima nel processo penale italiano? Revista Brasileira de Direito Processual Penal, 2019, n. 1, p. 73. https://doi.org/10.22197/ rbdpp.v4i1.130

BERTAGNA, Guido; CERETTI, Adolfo; MAZZUCCATO, Chiara. Il libro dell'incontro. Milano: Il Saggiatore, 2015.

BERTAGNINI, Elisa. Mediazione penale, giustizia riparativa e lavoro gratuito alla luce di qualche esperienza pratica. La legislazione penale, 2004, p. 401.

BERTOLINI, Benedetta. La messa alla prova per adulti sotto le lenti della giustizia riparativa. In: MARANDOLA, Antonella; LA REGINA, Katia; APRATI, Roberta (a cura di), Verso un processo penale accelerato, Napoli: Jovene, p. 25.

BRONZO, Pasquale. Il lavoro come fulcro del trattamento rieducativo. In: BRONZO, Pasquale; SIRACUSANO, Fabrizio; VICOLI, Daniele (a cura di), La riforma penitenziaria: novità e omissioni del nuovo "garantismo carcerario", Torino: Giappichelli, 2019, p. 117.

CARACENI, Lina. Voce "Processo penale minorile”. Enciclopedia del diritto. Aggiornamento, v. IV. Milano: Giuffrè, 2000, p. 1015.

CARNEVALE, Stefania. Accesso alle misure alternative. In: GIOSTRA, Glauco (a cura di), Carceri: materiali per la riforma. Disponibile in: www.archiviodpc. dirittopenaleuomo.org. Accesso in: 17.6.2015, p. 101.

CASTELLI, Stefano. La mediazione. Teorie e tecniche, Milano: Raffaello Cortina, 1996.

CATALANO, Elena Maria. La tutela della vittima nella direttiva 2012/29/UE e nella giurisprudenza delle corti europee. Rivista italiana di diritto e procedura penale, 2014, p. 1789.

CERETTI, Adolfo. Mediazione penale e giustizia. In: AA. VV., La mediazione penale in ambito minorile: applicazioni e prospettive, Milano: Franco Angeli, 1999, p. 99.

CESARI, Claudia. Le clausole di irrilevanza del fatto nel sistema processuale penale. Torino: Giappichelli, 2005.

CESARI, Claudia. Efficienza della giurisdizione penale e strategie di depenalizzazione processuale. In: KOSTORIS, Roberto E. (a cura di), La ragionevole durata del processo, Torino: Giappichelli, 2005, p. 95.

CESARI, Claudia. Commento all'art. 28. In: GIOSTRA, Glauco (a cura di), Il processo penale minorile, $2^{\mathrm{a}}$ ed., Milano: Giuffrè, 2007, p. 455. 
CESARI, Claudia. La Consulta supera le perplessità e la messa alla prova si radica nel sistema. Giurisprudenza costituzionale, 2018, p. 794.

CESARI, Claudia. La giustizia riparativa nel sistema penitenziario minorile: un nuovo orizzonte ancora incerto. In: CARACENI, Lina; COPPETTA Maria Grazia (a cura di), L'esecuzione delle pene nei confronti dei minorenni, Torino: Giappichelli, 2019, p. 47.

CIARDIELLO, Patrizia. Riparazione e mediazione nell'ambito dell'esecuzione penale per adulti. Rassegna penitenziaria e criminologica, 2007, n. 2, p. 95.

CIAVOLA, Agata. Il contributo della giustizia consensuale e riparativa all'efficienza dei modelli di giurisdizione. Torino: Giappichelli, 2010.

COLAMUSSI, Margherita. La messa alla prova. Padova: Cedam, 2010.

COMMISSIONE GIOSTRA. Il Progetto di riforma penitenziaria, Roma: Nuova Editrice Universitaria, 2019.

DELLA CASA, Franco. Misure alternative ed effettività della pena tra realtà e prospettive. Giustizia penale, 2001, II, c. 65.

DELLA CASA, Franco. Affidamento al servizio sociale o (pura e semplice) "pay-back sanction"? Equivoci sul significato dell'art. 47 co. 7 o.p. La legislazione penale, 2004, p. 380.

DI CHIARA, Giuseppe. La premura e la clessidra: i tempi della mediazione penale. Diritto penale e processo, 2015, p. 377.

DONINI, Massimo. Il delitto riparato. Una disequazione che può trasformare il sistema sanzionatorio. Diritto penale contemporaneo, Riv. trim., 2015, n. 2, p. 236.

FASSONE, Elvio, Voce "Probation e affidamento in prova”. Enciclopedia del diritto. Vol. XXXV. Milano: Giuffrè, 1986, p. 783.

FERRUA, Paolo. Una messa alla prova sul filo del rasoio costituzionale. In DANIELE, Marcello; PAULESU, Pier Paolo (a cura di), Strategie di deflazione processuale e rimodulazioni del giudizio in absentia, Torino: Giappichelli, 2016, p. 181.

FIORENTIN, Fabio. Uscito dalla porta, rientra dalla finestra l'obbligo del risarcimento del danno per la concessione dell'affidamento in prova del servizio sociale? Cassazione penale, 2009, p. 1200.

FIORENTIN, Fabio. Attività riparative. In: GIOSTRA, Glauco (a cura di), Carceri: materiali per la riforma. Disponibile in: www.archiviodpc.dirittopenaleuomo.org. Accesso in: 17.6.2015, p. 202.

GIORS, Barbara. Il lavoro all'esterno e gli incentivi volti a favorire l'attività lavorativa dei detenuti. In CAPRIOLI, Francesco; SCOMPARIN, Laura (a cura di), Sovraffollamento carcerario e diritti dei detenuti, Torino: Giappichelli, 2015, p. 143. 
GIOSTRA, Glauco; BRONZO, Pasquale (a cura di). Proposte per l'attuazione della delega penitenziaria. Roma: Sapienza Università Editrice, 2017.

GIOSTRA, Glauco, La riforma penitenziaria: il lungo e tormentato cammino verso la Costituzione. Diritto penale contemporaneo, 2018, n. 4, p. 119.

KILCHLING Michael; PARLATO, Lucia. Nuove prospettive per la restorative justice in seguito alla direttiva sulla vittima: verso un "diritto alla mediazione”? Germania e Italia a confronto. Cassazione penale, 2015, p. 4188.

LOGLI, Andrea, La sospensione del processo per messa alla prova tra equivoci dogmatici e limiti operativi. In DANIELE, Marcello; PAULESU, Pier Paolo (a cura di), Strategie di deflazione processuale e rimodulazioni del giudizio in absentia, Torino: Giappichelli, 2016, p. 133.

MAFFEO, Vania. I profili processuali della sospensione con messa alla prova. Napoli: Esi, 2017.

MAFFEO, Vania. Novità sistematiche in tema di messa alla prova. Per una riconsiderazione, costituzionalmente compatibile, del rapporto tra pena e processo. Cassazione penale, 2018, p. 3194.

MANNOZZI, Grazia. La giustizia senza spada, Giuffrè, Milano, 2003.

MANNOZZI, Grazia. La reintegrazione sociale del condannato tra rieducazione, riparazione ed empatia. Diritto penale e processo, 2012, p. 838.

MANNOZZI, Grazia. Le aperture alla giustizia riparativa nell'ambito delle misure alternative alla detenzione. Giurisprudenza italiana, 2016, p. 1530.

MANNOZZI, Grazia; LODIGIANI, Giovanni Angelo. La giustizia riparativa, Torino: Giappichelli, 2017.

MIRAGLIA, Michela. La messa alla prova dell'imputato adulto. Torino: Giappichelli, 2020.

MONTEVERDE, Lino. Mediazione e riparazione dopo il giudizio: l'esperienza della magistratura di sorveglianza. Minorigiustizia, 1999, n. 2, p. 86.

ORLANDI, Renzo. La mediazione penale tra finalità riconciliative ed esigenze di giustizia. In: AA. VV., Accertamento del fatto, alternative al processo, alternative nel processo, Milano: Giuffrè, 2007, p. 165.

PARISI, Francesco. La restorative justice alla ricerca di identità e legittimazione. Disponibile in: www.archiviodpc.dirittopenaleuomo.org. Accesso in: 24.12.2014, p. 1.

PARLATO, Lucia. La messa alla prova dopo il dictum della Consulta: indenne ma rivisitata e in attesa di nuove censure. Diritto penale contemporaneo, 2019, n. 1, p. 89. 
PATANÈ, Vania. La mediazione. In: GIOSTRA, Glauco; ILLUMINATI, Giulio (a cura di), Il giudice di pace nella giurisdizione penale, Torino: Giappichelli, 2000, p. 353. PATANÈ, Vania. Ambiti di attuazione di una giustizia conciliativa alternativa a quella penale: la mediazione. In: MESTITZ, Anna (a cura di), Mediazione penale: chi, dove, come e quando, Roma: Carrocci editore, 2004, p. 19.

SCOMPARIN, Laura. Quale giustizia riparativa dopo la conclusione del processo? La legislazione penale, 2004, p. 405.

UBERTIS, Giulio. Relazione. In AA. VV., Accertamento del fatto, alternative al processo, alternative nel processo, Milano: Giuffrè, 2007, p. 143.

VICOLI, Daniele. Il ruolo della vittima nella fase esecutiva tra occasioni mancate e scenari futuri. In BARGIS, Marta; BELLUTA, Hervé (a cura di), Vittime di reato e sistema penale. Torino: Giappichelli, 2017, p. 527.

\section{Additional information and author's declarations (scientific integrity)}

Conflict of interest declaration: the author confirms that there are no conflicts of interest in conducting this research and writing this article.

Declaration of authorship: all and only researchers who comply the authorship requirements of this article are listed as authors; all coauthors are fully responsible for this work in its entirety.

Declaration of originality: the author assures that the text here published has not been previously published in any other resource and that future republication will only take place with the express indication of the reference of this original publication; he also attests that there is no third party plagiarism or self-plagiarism. 


\section{Editorial process dates}

(http://www.ibraspp.com.br/revista/index.php/RBDPP/about/editorialPolicies)

- Submission: 22/07/2021

- Desk review and plagiarism check: 02/08/2021

- Review 1: 10/08/2021

- Review 2: 19/08/2021

- Preliminary editorial decision: $20 / 08 / 2021$

- Correction round return: 02/09/2021

- Final editorial decision: 02/09/2021

\section{Editorial team}

- Editor-in-chief: 1 (VGV)

- Associated-editor: 1 (BC)

- Reviewers: 2

\section{HOW TO CITE (ABNT BRAZIL):}

VICOLI, Daniele. La mediazione in fase esecutiva nel sistema italiano: il quadro normativo e le dinamiche applicative. Revista Brasileira de Direito Processual Penal, vol. 7, n. 3, p. 2285-2310, set./ dez. 2021. https://doi.org/10.22197/rbdpp.v7i3.623

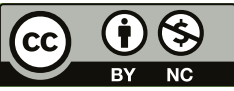

